\title{
MRI Predictors of Recurrence and Outcome after Acute Transverse Myelitis of Unidentified Etiology
}

\author{
(DE. Bulut, (D) T. Shoemaker, (D). Karakaya, (DD.M. Ray, (D) M.A. Mealy, (D). Levy, and (DI. Izbudak
}

\begin{abstract}
BACKGROUND AND PURPOSE: The early prediction of recurrence after an initial event of transverse myelitis helps to guide preventive treatment and optimize outcomes. Our aim was to identify MR imaging findings predictive of relapse and poor outcome in patients with acute transverse myelitis of unidentified etiology.
\end{abstract}

MATERIALS AND METHODS: Spinal MRIs of 77 patients (mean age, $36.3 \pm 20$ years) diagnosed with acute transverse myelitis were evaluated retrospectively. Only the patients for whom an underlying cause of myelitis could not be identified within 3 months of symptom onset were included. Initial spinal MR images of patients were examined in terms of lesion extent, location and distribution, brain stem extension, cord expansion, $\mathrm{T} 1$ signal, contrast enhancement, and the presence of bright spotty lesions and the owl's eyes sign. The relapse rates and Kurtzke Expanded Disability Status Scale scores at least 1 year (range, 1-14 years) after a myelitis attack were also recorded. Associations of MR imaging findings with clinical variables were studied with univariate associations and binary log-linear regression. Differences were considered significant for $P$ values $<.05$.

RESULTS: Twenty-seven patients (35.1\%) eventually developed recurrent disease. Binary logistic regression revealed 3 main significant predictors of recurrence: cord expansion (OR, 5.30; 95\% Cl, 1.33-21.11), contrast enhancement (OR, 5.05; 95\% Cl, 1.25-20.34), and bright spotty lesions (OR, 3.63; $95 \% \mathrm{Cl}, 1.06-12.43)$. None of the imaging variables showed significant correlation with the disability scores.

CONCLUSIONS: Cord expansion, contrast enhancement, and the presence of bright spotty lesions could be used as early MR imaging predictors of relapse in patients with acute transverse myelitis of unidentified etiology. Collaborative studies with a larger number of patients are required to validate these findings.

ABBREVIATIONS: BSL = bright spotty lesion; EDSS = Expanded Disability Status Scale; LETM = longitudinally extensive transverse myelitis; NMOSD = neuromyelitis optica spectrum disorder

l: the initial evaluation of acute transverse myelitis, a specific underlying cause of the immunologic attack cannot be determined in many circumstances. The early prediction of recurrence in such cases helps to guide preventive treatment, which, in turn, may improve long-term prognosis. Alternatively, features that favor monophasic disease may save the patients from unnecessary

Received April 6, 2019; accepted after revision June 6.

From the Departments of Radiology (E.B.) and Statistics (J.K.), Hacettepe University Faculty of Medicine, Ankara, Turkey; Department of Neurology (T.S., M.A.M., M.L.) and Division of Neuroradiology (D.M.R., I.I.), The Russell H. Morgan Department of Radiology and Radiological Science, Johns Hopkins University School of Medicine, Baltimore, Maryland; Department of Neurological Sciences (T.S.), Rush University Medical Center, Chicago, Illinois; and Department of Neurology (M.L.), Massachusetts General Hospital and Harvard Medical School, Boston, Massachusetts.

This study was funded by a Research Project Grant from the National Institute of Allergy and Infectious Diseases, grant Al130548, ML.

Paper previously presented, in part, as an oral presentation at: American Society of Neuroradiology Annual Meeting and the Foundation of the ASNR Symposium, June 2-7, 2018; Vancouver, British Columbia, Canada. The presentation number was O-257. long-term immunosuppression. Accordingly, several independent demographic and laboratory risk factors for recurrent disease after acute transverse myelitis have been determined, including female sex, African American race, vitamin D insufficiency, and serum antibodies such as anti-aquaporin 4 antibodies, antiRo/SS-A antibodies, and a high ( $\geq 1: 160)$ antinuclear antibody titer. ${ }^{1}$ Previous studies also suggested clinical factors associated with poor functional recovery, such as symmetric motor dysfunction at onset, sphincter dysfunction, and spinal cord shock-like symptoms. $^{2,3}$

Unlike previously established clinical variables, imaging predictors of relapse and poor outcome after acute transverse myelitis of unidentified etiology have not been comprehen-

\footnotetext{
Please address correspondence to Elif Bulut, MD, Hacettepe University Faculty of Medicine, Department of Radiology, Sihhiye Ankara, Turkey; e-mail: drelifbulut@yahoo.com

- Indicates open access to non-subscribers at www.ajnr.org

http://dx.doi.org/10.3174/ajnr.A6121
} 


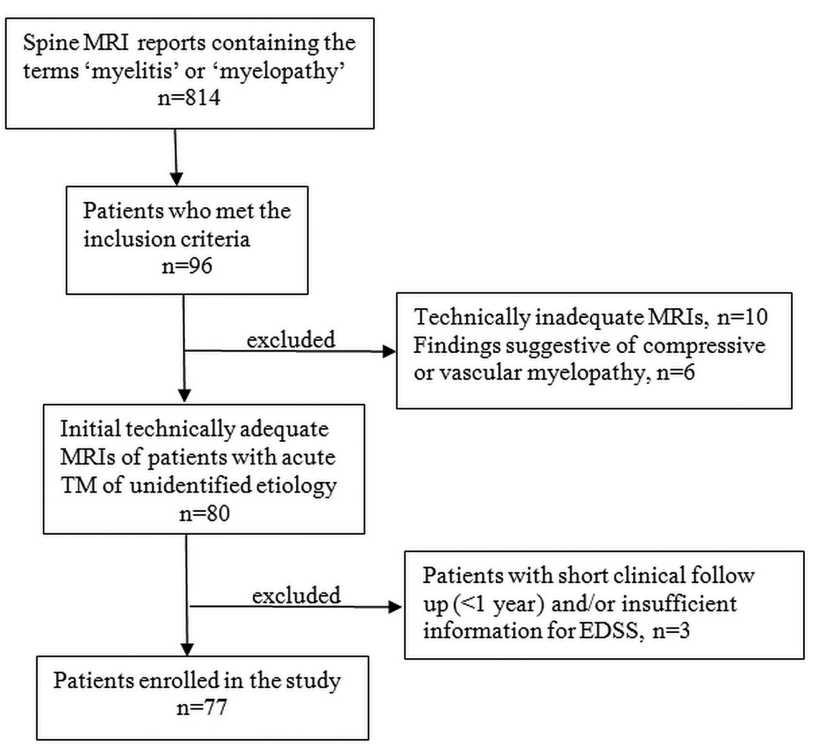

FIG 1. Flow chart of patients who met the inclusion/exclusion criteria for the study population.

sively studied to date. Longitudinally extensive lesions extending $\geq 3$ vertebral lengths have been associated with the relapsing myelitis diseases, neuromyelitis optica, and myelin oligodendrocyte glycoprotein antibody disease. ${ }^{1}$ In addition, there are other MR imaging features that could be used to predict relapse or outcome, including contrast enhancement, expansion, bright spotty lesions, and T1 hypointensity, that have not been assessed in this patient population. Therefore, we aimed to identify spinal MR imaging findings at onset that are associated with an increased risk of recurrence and poor outcome.

\section{MATERIALS AND METHODS}

An institutional review board-approved data base search was performed using the keywords "myelitis" and "myelopathy" in spine MR imaging reports dictated at Johns Hopkins Hospital from January 1, 2005, to January 1, 2017. The acquired MR imaging reports were reviewed with clinical data to identify patients referred for the diagnosis of acute transverse myelitis. The inclusion criteria were as follows: 1) fulfillment of diagnostic criteria established by the Transverse Myelitis Consortium Working Group 2002 for acute transverse myelitis ${ }^{4}$;2) patients whose initial evaluation (within 3 months of symptom onset) could not yield sufficient information to identify a specific underlying disorder (eg, aquaporin-4 antibody testing negative for neuromyelitis optica); and 3) patients whose spinal MR imaging studies were obtained within 1 month of symptom onset. ${ }^{5}$ The patients with technically inadequate scans or having MR imaging findings of a compressive etiology or abnormal flow voids on the cord surface, consistent with vascular myelopathy, were excluded (Fig 1).

Patient demographics, serologies, and relapse rates were recorded. The Kurtzke Expanded Disability Status Scale (EDSS) scores ${ }^{6}$ at least 1 year (range, 1-14 years) after the first attack were assigned retrospectively using clinical documentation. Relapsing disease was defined as a clinical relapse with a change on neurologic examination and a report of a new lesion on spine MR imaging. Patients with short clinical follow-up
$(<1$ year $)$ and/or insufficient information for calculating the EDSS were further excluded from the study (Fig 1).

Spinal cord MR imaging studies were performed at presentation, before treatment (mean, 10 days after symptom onset; range, 1-30 days) either on $1.5 \mathrm{~T}$ or 3T magnets from different manufacturers: Achieva (Philips Medical Systems, Best, the Netherlands), Signa Excite (GE Healthcare, Milwaukee, Wisconsin), and Aera, Avanto, Verio, Skyra and Trio (Siemens, Erlangen, Germany). For some patients, the initial MR imaging study was performed at an outside institution before the transfer to our medical center. Spinal MR imaging protocol included sagittal T1-weighted, sagittal and axial T2-weighted, sagittal STIR, and contrast-enhanced sagittal and axial T1-weighted imaging $(n=76)$. The details of the acquisition parameters are provided in Table 1.

Image analysis was performed in consensus by 2 neuroradiologists (E.B. and I.I.) blinded to the clinical outcome. T2-hyperintense spinal cord lesions were evaluated in terms of the following: 1) extent, 2) location, 3) distribution, 4) brain stem extension, 5) cord expansion (ie, swelling), 6) T1 signal, and 7) contrast enhancement.

The extent of involvement, lesion distribution, and cord expansion were evaluated from sagittal T2-weighted images. The lesions were grouped as either longitudinally extensive or shortsegment lesions according to the number of the vertebral bodies spanned. The lesions extending at least 3 vertebral levels were considered longitudinally extensive lesions. According to the cord segments involved, lesion distribution was grouped as cervical, cervicothoracic, thoracic, or confined to the conus. Lesion location was determined from axial T2-weighted images and classified as lesions confined to gray matter, confined to white matter, or involving both gray and white matter. Lesions involving $\geq 50 \%$ of the spinal cord area were also noted, as were multiple lesions with no radiologically detectable continuity. The presence of bright spotty lesions (lesions similar to or higher in signal intensity than CSF) and the owl's eyes sign (Fig 2$)^{7}$ were also assessed from axial T2-weighted images. The presence and pattern of contrast enhancement were assessed on both axial and sagittal postcontrast images of available examinations.

When available, brain MR imaging examinations performed at the same time or within 1 month of the spine MR imaging were also reviewed. The brain MR imaging protocol included T1weighted images, fast spin-echo T2-weighted images, FLAIR images, diffusion-weighted images, and postcontrast T1-weighted images. The brain MR imaging findings were classified as either normal/insignificant or abnormal. The findings were classified as "abnormal" in the following circumstances: multiple $(\geq 2)$ nonspecific lesions not fulfilling the 2017 McDonald MR imaging criteria; extensive cerebral lesions with or without contrast enhancement; findings suggestive of neuromyelitis optica spectrum disorder (NMOSD) but not sufficient to fulfill the diagnostic criteria, such as lesions located in the hypothalamus or area postrema; and leptomeningeal enhancement.

\section{Statistical Analysis}

Demographic data, serologies, and MR imaging findings were analyzed for associations with recurrence and EDSS. EDSS scores of patients with longitudinally extensive transverse myelitis (LETM) 
Table 1: Imaging parameters used in spinal MRI

\begin{tabular}{lclccc}
\hline \multicolumn{1}{c}{ Sequence } & TR/TI $(\mathbf{m s})$ & TE $(\mathbf{m s})$ & Matrix Size & FOV $(\mathbf{m m})$ & Section Thickness/Spacing $(\mathbf{m m})$ \\
\hline TIWI sagittal & $397-562$ & $8.7-11$ & $256-384 \times 256-288$ & $25-32 \times 35.3-45.2$ & $3-3.5 / 3.3-4.3$ \\
T2WI sagittal & $2340-4070$ & $96-109$ & $256-384 \times 256-288$ & $25-32 \times 35.3-45.2$ & $3-3.5 / 3.3-4.3$ \\
STIR sagittal & $3210-4610 / 150-220$ & $42-79$ & $256-384 \times 256-288$ & $25-32 \times 35.3-45.2$ & $3-3.5 / 3.3-4.3$ \\
T2WI axial & $2874-4100$ & $98-109$ & $256-320 \times 192-280$ & $18-20 \times 25.4-28.3$ & $3-4 / 3.3-5$ \\
TIWI axial & $400-654$ & $8.4-9.7$ & $256-320 \times 192-280$ & $18-20 \times 25.4-28.3$ & $3-4 / 3.3-5$ \\
\hline
\end{tabular}

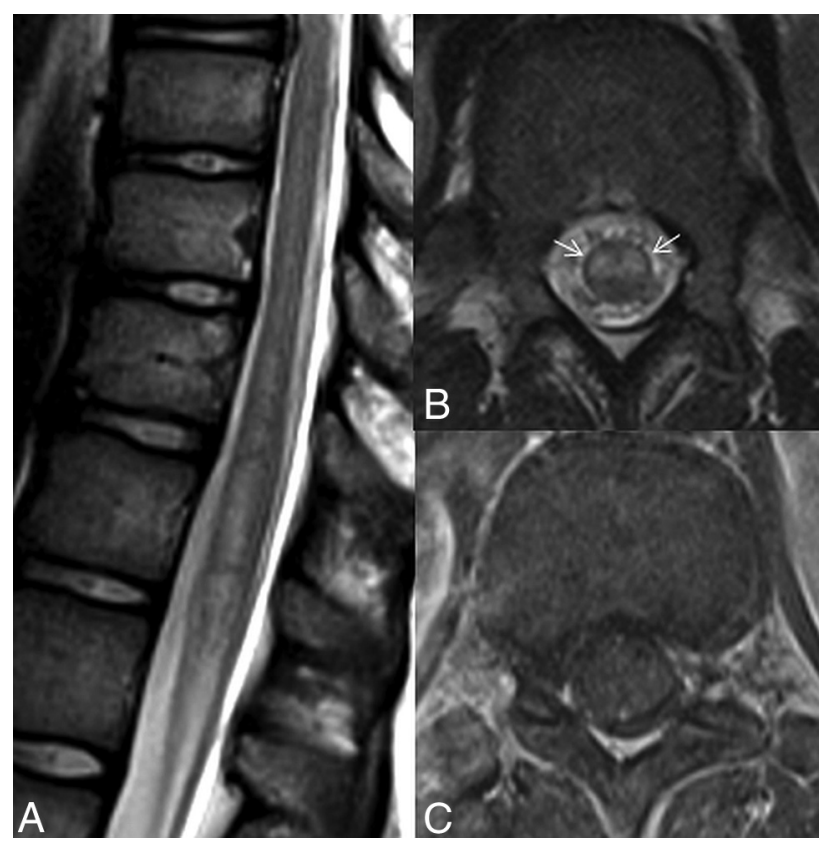

FIG 2. Spine MR images of a 13-year-old female patient with monophasic idiopathic transverse myelitis. The sagittal T2-weighted image (A) shows a longitudinally extensive, minimally expansile hyperintense lesion at the distal spinal cord. The axial T2-weighted $(B)$ and postcontrast Tl-weighted $(C)$ images show central involvement with the owl's eyes sign (arrows in $B$ ) and no discernible contrast enhancement of the lesion.

were also compared with those having short-segment lesions involving $\leq 50 \%$ of the transectional area of the cord and with those of patients having short-segment lesions confined to the white matter. All analyses were performed with the SPSS (Version 23.0; IBM, Armonk, New York) statistical package program. Numeric variables were evaluated for normality of data distribution using the Kolmogorov-Smirnov test. Descriptive statistics were expressed as mean \pm SD or median (minimum-maximum), according to the assumption of normal distribution for quantitative variables. Qualitative variables were given as proportions (percentages). An independent-samples $t$ test was performed to compare the means of 2 independent groups. For non-normally distributed data, the Mann-Whitney $U$ test was used to compare the 2 groups. The $\chi^{2}$ (continuity correction or Fisher exact test) was used to compare differences among groups for categoric variables. A $P$ value $<.05$ was accepted as statistically significant.

Logistic regression analysis was performed to determine important risk factors for developing recurrent myelitis. Binary logistic regression with a backward stepwise method was used to evaluate which independent imaging variables were statistically significant predictors of the binary dependent variable (relapse).
Using the logistic models, we calculated ORs and their respective $95 \%$ confidence intervals.

\section{RESULTS}

Initial inquiry of spine MR imaging reports containing the terms "myelitis" or "myelopathy" yielded 814 MR imaging scans. After we applied inclusion and exclusion criteria, 77 patients (female/ male ratio, 46:31; mean age, $36.4 \pm 20$ years) were enrolled in the study. The clinical characteristics of the patients are provided in Table 2 . Twenty-seven patients (35.1\%) eventually developed recurrent disease. The mean time to relapse was 17.3 months (range, $3-117$ months). Time to relapse was $\leq 1$ year in 21 patients $(77 \%)$. Eighteen $(66.6 \%)$ patients had $>1$ relapse (mean, 3.05; range, $2-5$ relapses). Age at presentation did not differ between patients with monophasic and recurrent disease $(P=.2)$. There were higher ratios of female $(P=.03)$ and African American patients $(P=.02)$ in the recurrent group, as expected. Among the 27 patients who relapsed, 8 patients (29.6\%) eventually seroconverted for the antiaquaporin 4 antibody after initially testing negative, all of whom presented with LETM at onset. Four patients with relapse were ultimately diagnosed with aquaporin 4 seronegative NMOSD, 3 with multiple sclerosis, 1 with acute disseminated encephalomyelitis, 1 with neurosarcoidosis, 1 with systemic lupus erythematosus, and the other 9 (33\%) were diagnosed with recurrent transverse myelitis of unknown etiology. The mean time to diagnosis was 23.2 months (range, 3.2-108 months) for the 18 patients with recurrence and specific diagnoses. For the 9 patients with recurrent transverse myelitis of unknown etiology, the mean follow-up time was 45.2 months (range, 13.5-169 months), which was much longer than the mean time to diagnosis in patients with specific diagnoses.

The frequencies of spinal cord MR imaging findings and associations with monophasic/relapsing disease are outlined in Table 3. Imaging findings of LETM, brain stem extension, cord expansion, bright spotty lesions (BSLs), and contrast enhancement were higher among patients who relapsed (Fig 3). When binary logistic regression with the backward stepwise method was used, findings of cord expansion, BSLs, and contrast enhancement were calculated to be significant predictors of recurrent disease (Table 4).

Thirteen (48\%) of the patients having BSLs on their spinal MR imaging were ultimately diagnosed with NMOSD. Multiple sclerosis $(n=1)$, acute disseminated encephalomyelitis $(n=1)$, and neurosarcoidosis $(n=1)$ were other entities with BSLs. Additional MR imaging features of the NMOSD cohort included cord expansion and brain stem extension.

Brain MR imaging was available in 63 patients, of whom 17 had abnormal findings not necessarily suggestive of a specific diagnosis, except for 1 patient with NMOSD who had a lesion in the area postrema. Two patients had cerebral leptomeningeal enhancement without a discernible parenchymal lesion. 
Table 2: The clinical characteristics of 77 patients who presented with acute transverse myelitis

\begin{tabular}{|c|c|c|c|}
\hline Characteristics & $\begin{array}{c}\text { Monophasic } \\
(n=50)\end{array}$ & $\begin{array}{l}\text { Recurrent } \\
(n=27)\end{array}$ & $\begin{array}{c}P \\
\text { Values }\end{array}$ \\
\hline Age at initial manifestation (mean) (yr) & $34.2 \pm 20.6$ & $40.4 \pm 18.5$ & .196 \\
\hline Female $(n)(\%)$ & $25(50 \%)$ & $21(77.8 \%)$ & .033 \\
\hline Ethnicity & & & .020 \\
\hline African American ( $n)(\%)$ & $10(20 \%)$ & $10(37 \%)$ & \\
\hline Caucasian $(n)(\%)$ & $38(76 \%)$ & $12(44.4 \%)$ & \\
\hline Asian American (n) (\%) & $1(2 \%)$ & $3(11 \%)$ & \\
\hline Hispanic $(n)(\%)$ & $1(2 \%)$ & $2(7.4 \%)$ & \\
\hline Seropositivity for anti-AQP4 Ab & $1(2 \%)$ & $8(29.6 \%)$ & .003 \\
\hline Mean follow-up time (yr) & $2.4 \pm 1.7$ & $3.8 \pm 3.8$ & .023 \\
\hline Mean EDSS score & $4.2 \pm 2.1$ & $3.3 \pm 1.8$ & .064 \\
\hline Patients with follow-up spine MRIs ( $n$ ) (\%) & $16(32 \%)$ & $27(100 \%)$ & $<.001$ \\
\hline $\begin{array}{l}\text { Use of immunosuppressive/immunomodulatory } \\
\text { treatment }(n)(\%)\end{array}$ & $9(18 \%)$ & $18(66.7 \%)$ & $<.001$ \\
\hline
\end{tabular}

Note:-AQP4 Ab indicates aquaporin 4 antibody.

Table 3: The frequencies of spinal MRI findings and associations with monophasic/relapsing disease

\begin{tabular}{|c|c|c|c|c|}
\hline MRI Findings & $\begin{array}{c}\text { Monophasic Disease } \\
(n=50)(n)(\%)\end{array}$ & $\begin{array}{c}\text { Recurrent Disease } \\
(n=27)(n)(\%)\end{array}$ & $\begin{array}{c}P \\
\text { Value }\end{array}$ & $\begin{array}{l}\text { Unadjusted } \\
\text { OR }(95 \% \mathrm{CI})\end{array}$ \\
\hline $\operatorname{LETM}(n=42)(54.5 \%)$ & $22(44 \%)$ & $20(74.1 \%)$ & $.022^{\mathrm{a}}$ & $3.63(1.30-10.14)$ \\
\hline Multifocal lesions $(n=16)(22.5 \%)$ & $9 / 44(20.5 \%)$ & $7(25.9 \%)$ & .808 & $1.36(0.44-4.21)$ \\
\hline Distribution & & & .304 & \\
\hline Cervical $(n=21)(27.3 \%)$ & $14(28 \%)$ & $7(25.9 \%)$ & & \\
\hline Cervicothoracic $(n=14)(18.2 \%)$ & $6(12 \%)$ & $8(29.6 \%)$ & & \\
\hline Thoracic $(n=35)(45.5 \%)$ & $25(50 \%)$ & $10(37 \%)$ & & \\
\hline Holocord $(n=7)(9.1 \%)$ & $5(10 \%)$ & $2(7.4 \%)$ & & \\
\hline Brain stem extension $(n=5)(6.5 \%)$ & $1 / 46(2.2 \%)$ & $4 / 25(16 \%)$ & $.049^{\mathrm{a}}$ & $8.57(0.90-81.46)$ \\
\hline Location & & & .521 & \\
\hline Gray matter $(n=3)(3.9 \%)$ & $3(6 \%)$ & 0 & & \\
\hline Gray + white matter $(n=65)(84.4 \%)$ & $42(84 \%)$ & $23(85.2 \%)$ & & \\
\hline White matter $(n=9)(11.7 \%)$ & $5(10 \%)$ & $4(14.8 \%)$ & & \\
\hline$>1 / 2$ of the cord area $(n=55)(71.4 \%)$ & $33(66 \%)$ & $22(81.5 \%)$ & .242 & $2.26(0.73-7.04)$ \\
\hline Cord expansion $(n=48)(62.3 \%)$ & $26(52 \%)$ & $22(81.5 \%)$ & $.021^{\mathrm{a}}$ & $4.06(1.32-12.42)$ \\
\hline TT hypointensity $(n=23)(30.3 \%)$ & $11 / 49(22.4 \%)$ & $12(44.4 \%)$ & .089 & $2.76(1.01-7.61)$ \\
\hline BSLs $(n=27)(35.1 \%)$ & $12(24 \%)$ & $15(55.6 \%)$ & $.012^{\mathrm{a}}$ & $3.95(1.45-10.74)$ \\
\hline Owl's eyes sign $(n=2)(2.6 \%)$ & $2(4 \%)$ & 0 & .539 & $0.58(0.06-5.88)$ \\
\hline Enhancement $(n=48)(62.3 \%)$ & $26(52 \%)$ & $22 / 26(84.6 \%)$ & $.011^{\mathrm{a}}$ & $5.07(1.52-16.87)$ \\
\hline Brain involvement $(n=17)(27 \%)$ & $11 / 43(25.6 \%)$ & $6 / 20(30 \%)$ & .950 & $1.24(0.38-4.04)$ \\
\hline
\end{tabular}

${ }^{a}$ Significant.

There was no significant correlation between recurrent disease and EDSS scores of the patients $(P=.14)$, though there was an unexpected trend for recurrent cases to end up with better EDSS scores, at least early in their disease course. Also, none of the imaging variables analyzed for association with recurrent disease showed significant correlation with EDSS scores. We could not find a significant difference between EDSS scores of the patients with LETM and scores of patients having short-segment lesions involving $\leq 50 \%$ of the transectional area of the cord or confined to white matter.

\section{DISCUSSION}

Spinal cord MR imaging is essential in the evaluation of acute transverse myelitis because MR imaging features, including location, distribution and extent of the lesions, and enhancement pattern could narrow the differential diagnosis considerably. Identification of MR imaging features predictive of relapsing disease may also prompt clinical decision-making in favor of empiric treatment with immunosuppression. These patients also could be considered candidates for more frequent follow-up within the first year of the initial attack with repeat serologic testing. This consideration is because titers of specific antibodies, such as the anti-aquaporin 4 antibody, may fluctuate and retesting may reveal sufficient levels to confirm a diagnosis of NMOSD.

There was a significantly higher rate of recurrent myelitis in female and African American patients in our cohort. This finding was similar to findings in the previous report of Kimbrough et al, ${ }^{1}$ and most likely reflects a higher incidence of NMOSD in these demographic groups. Although it is often associated with NMOSD, LETM has a wide range of differential diagnoses, including parainfectious myelitis, systemic autoimmune disease-related myelitis, and idiopathic transverse myelitis. ${ }^{8}$ A significantly higher proportion of patients with recurrent disease in our cohort had LETM, which was compatible with the previous reports noting the increased risk of developing recurrent disease in LETM. ${ }^{1,9}$

As distinctive features of our study, cord expansion, BSLs, and enhancement were also found promising for predicting relapse after acute transverse myelitis at initial evaluation. Cord expansion was previously suggested to be specific to NMOSD and useful in differentiating it from longitudinally extensive lesions of MS. ${ }^{10-12}$ On the other hand, cord expansion did not significantly 


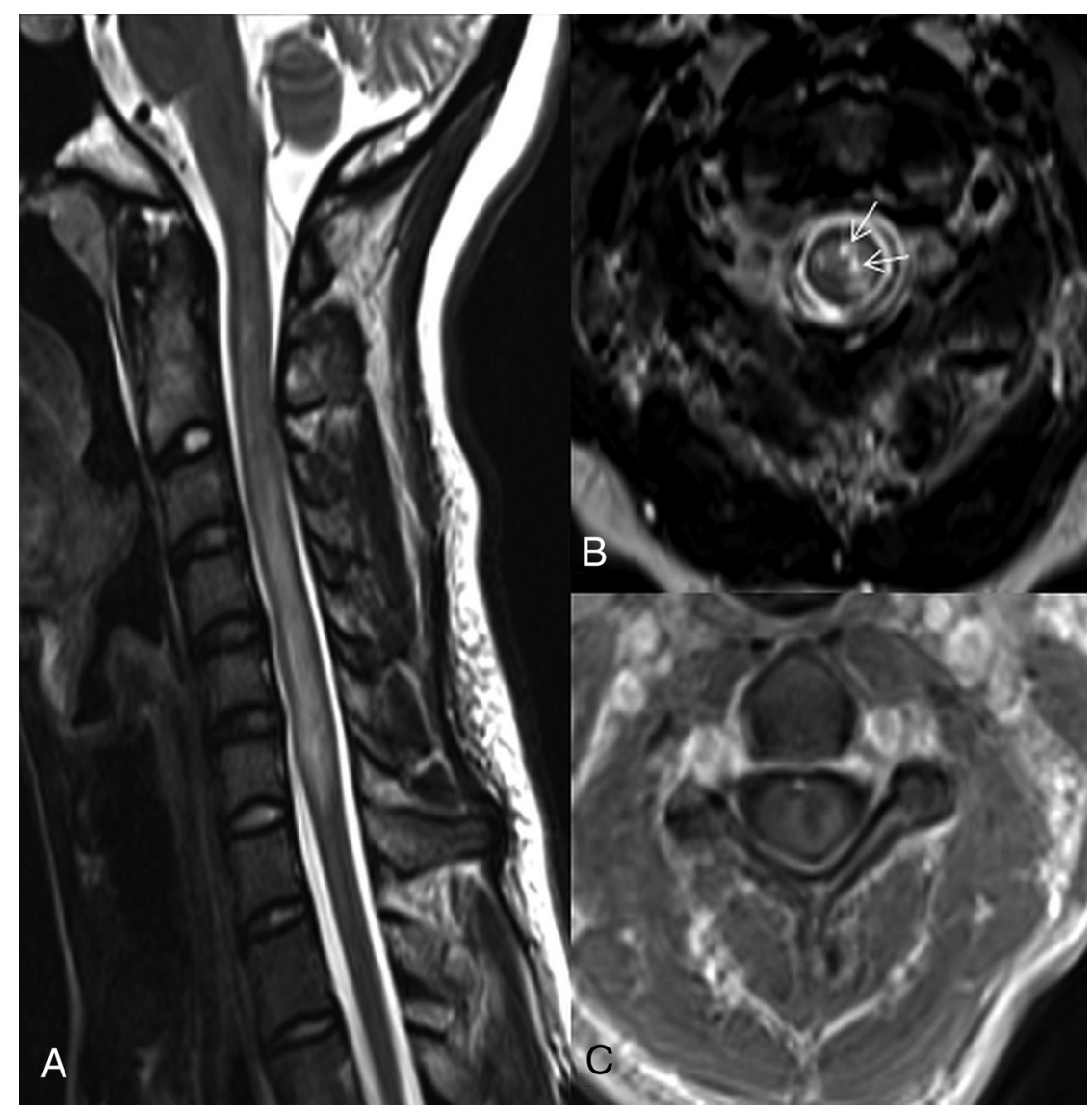

FIG 3. Spine MR images of a 29-year-old female patient with recurrent transverse myelitis. The sagittal T2-weighted image ( $A$ ) shows a longitudinally extensive, expansile hyperintense lesion at the cervical spine. The axial T2-weighted $(B)$ and postcontrast T1-weighted $(C)$ images show gray and white matter involvement with bright spotty lesions (arrows in $B$ ) and ring enhancement of the lesion. The patient was diagnosed with NMOSD later at follow-up.

Table 4: Adjusted ORs for associations of imaging variables/age

\begin{tabular}{lccc}
\hline \multicolumn{1}{c}{ Variable } & $\begin{array}{c}\text { Adjusted } \\
\text { ORs }\end{array}$ & $\begin{array}{c}\boldsymbol{P} \\
\text { Value }\end{array}$ & $\mathbf{9 5 \% ~ C l}$ \\
\hline Cord expansion & 5.30 & .018 & $1.33-21.11$ \\
BSLs & 3.63 & .040 & $1.06-12.43$ \\
Enhancement & 5.05 & .023 & $1.25-20.34$ \\
Age & 1.03 & .084 & $0.99-1.061$ \\
Constant & 0.01 & .00 & \\
\hline
\end{tabular}

distinguish neuromyelitis optica from other causes of LETM in another recent study. ${ }^{5}$ Most cases showing cord expansion were non-NMOSD in our cohort. This could be explained by the low number of previously diagnosed cases of acute NMOSD in our study, which were excluded.

BSLs are defined as hyperintense small lesions on T2-weighted images, similar to or higher in signal intensity than CSF. ${ }^{13}$ They have been previously suggested as a highly distinctive feature of NMOSD and infrequently noted in multiple sclerosis and other causes of LETM. ${ }^{5,13}$ Similarly, a large number of our patients with BSLs (48\%) were later diagnosed with NMOSD, though cases that remained idiopathic were also represented. The predictive value of both bright spotty lesions and cord expansion is likely driven by their association with NMOSD.

Brain stem extension was an infrequent finding $(n=5)$ in our cohort. Although infrequent, brain stem extension has been re- ported as specific to NMOSD. ${ }^{10,12}$ Similarly, most of our patients (75\%) with brain stem extension had a final diagnosis of NMOSD. Although it was found to increase the risk of recurrence in univariate analysis, adjusted odds ratios were not significant. This is probably due to its low frequency, which could affect the statistical analysis.

Although it partially defines acute attacks of inflammatory myelitis, contrast enhancement on MR imaging could also occur in other etiologies, including dural arteriovenous fistula and spondylotic myelopathy. ${ }^{8}$ It was reported to be more frequent in multiple sclerosis and other autoimmune etiologies compared with idiopathic myelitis in a previous study. ${ }^{14}$ Contrast enhancement, regardless of its pattern, was also found to be significantly higher in NMOSD compared with the other causes of LETM. ${ }^{5}$ It was a frequent finding in our cohort, with a wide range of patterns, from patchy or nodular to ring enhancement. We found a significant correlation between contrast enhancement and the risk of developing relapse, reflecting the greater incidence of contrast enhancement in disease entities associated with increased relapse rates. Our result is different from those in previous reports that could not find a significant association between enhancement and recurrence. This difference could be explained by small cohort size $e^{9}$ and differences in case selection ${ }^{3}$ in those studies.

Brain MR imaging features could give a clue to recognize a specific etiology in an acute transverse myelitis attack. For example, brain lesions localized at sites rich in aquaporin 4 expression such as the area postrema are highly suggestive of NMOSD. Brain MR imaging findings either nonspecific or suggestive of a specific etiology were not frequent in our cohort, probably due to preclusion of patients who could be diagnosed at initial evaluation. The low frequency of brain lesions could negatively affect the statistical power of its association with recurrence or outcome.

Recurrent disease was not associated with poor outcome in our study, similar to a previous study that analyzed prognostic factors in LETM. ${ }^{9}$ This finding is probably due to the relatively short mean follow-up in both studies, because in another study ${ }^{3}$ with longer follow up (mean, 6.2 years), relapsing disease was found to be a predictor of worse outcome.

Previous studies yielded conflicting results regarding the association of LETM and functional outcome..$^{2,9,14,15}$ Contrary to the studies of Carnero Contentti et al, ${ }^{2,15}$ LETM did not predispose to poor functional outcome in our cohort. This variation may be due to differences in patient groups. The partial lesions defined in the study of Carnero Contentti et al, ${ }^{15}$ which are frequently associated with multiple sclerosis, were uncommon $(n=8)$ in our study. 
Additionally, the mean follow-up period of our study is much longer than those in 2 previous studies ( 3 and 3-6 months). Our results were similar to those in prior studies with longer follow-up periods. $^{9,14}$

Other spinal MR imaging features that we analyzed (distribution, T1 signal, extension in a transverse section, multiplicity, and contrast enhancement) were also evaluated in a previous study in which no association could be found with clinical outcome after acute transverse myelitis. ${ }^{16}$ Our study supports these findings, though more studies with large numbers of cases are needed for confirmation.

This retrospective study has several limitations. Most important, the follow-up periods varied widely, and we cannot rule out a future relapse in all of the patients who are currently monophasic.

Additionally, most patients with monophasic disease in our cohort did not receive preventive immunosuppression treatment during follow-up; therefore, the effects of those therapies on outcome could not be estimated. Other limitations were related to image acquisition. As a retrospective study, MR images were obtained from scanners with different magnet strengths and different sequence parameters. Also, postcontrast images were not acquired in a patient. All of these limitations could affect the sensitivity of image evaluation.

\section{CONCLUSIONS}

MR imaging findings of cord expansion, BSLs, and contrast enhancement could be used as early imaging predictors of relapse in acute transverse myelitis of unidentified etiology. Collaborative studies with larger number of patients are required to validate these findings.

Disclosures: Michael Levy—RELATED: Grant: Transverse Myelitis Association, Comments: grant to study genetics of transverse myelitis*; UNRELATED: Board Membership: Genentech, Alexion Pharmaceuticals, and Viela Bio; Consultancy: Genentech, Alexion Pharmaceuticals, and Viela Bio, Gerson Lehrman Group; Expert Testimony: various legal firms; Grants/Grants Pending: National Institutes of Health, Alexion Pharmaceuticals, Alnylam Pharmaceuticals, Viela Bio*; Travel/Accommodations/ Meeting Expenses Unrelated to Activities Listed: Alexion Pharmaceuticals, Genentech, Union Chimique Belge Pharma. Izlem Izbudak-UNRELATED: Consultancy: Alexion Pharmaceuticals, Comments: MRI reads for neuromyelitis optica recurrence adjudication; Grants/Grants Pending: Biogen, Comments: MSPATHS study.* *Money paid to the institution.

\section{REFERENCES}

1. Kimbrough DJ, Mealy MA, Simpson A, et al. Predictors of recurrence following an initial episode of transverse myelitis. Neurol Neuroimmunol Neuroinflamm 2014;1:e4 CrossRef Medline
2. Carnero Contentti E, Hryb JP, Leguizamón F, et al. Differential diagnosis and prognosis for longitudinally extensive myelitis in Buenos Aires, Argentina [in Spanish, English]. Neurologia 2017;32:99105 CrossRef Medline

3. Gajofatto A, Monaco S, Fiorini M, et al. Assessment of outcome predictors in first-episode acute myelitis: a retrospective study of 53 cases. Arch Neurol 2010;67:724-30 CrossRef Medline

4. Transverse Myelitis Consortium Working Group. Proposed diagnostic criteria and nosology of acute transverse myelitis. Neurology 2002;59:499-505 CrossRef Medline

5. Pekcevik Y, Mitchell CH, Mealy MA, et al. Differentiating neuromyelitis optica from other causes of longitudinally extensive transverse myelitis on spinal magnetic resonance imaging. Mult Scler 2016;22:302-11 CrossRef Medline

6. Kurtzke JF. Rating neurologic impairment in multiple sclerosis: an expanded disability status scale (EDSS). Neurology 1983;33:1444-52 CrossRef Medline

7. Lebouteux MV, Franques J, Guillevin R, et al. Revisiting the spectrum of lower motor neuron diseases with snake eyes appearance on magnetic resonance imaging. Eur J Neurol 2014;21:1233-41 CrossRef Medline

8. Flanagan EP. Autoimmune myelopathies. Handb Clin Neurol 2016; 133:327-51 CrossRef Medline

9. Sepúlveda M, Blanco Y, Rovira A, et al. Analysis of prognostic factors associated with longitudinally extensive transverse myelitis. Mult Scler 2013;19:742-48 CrossRef Medline

10. Cassinotto C, Deramond H, Olindo S, et al. MRI of the spinal cord in neuromyelitis optica and recurrent longitudinal extensive myelitis. J Neuroradiol 2009;36:199-205 CrossRef Medline

11. Qiu W, Wu JS, Zhang MN, et al. Longitudinally extensive myelopathy in Caucasians: a West Australian study of 26 cases from the Perth Demyelinating Diseases Database. J Neurol Neurosurg Psychiatry 2010;81:209-12 CrossRef Medline

12. Kitley JL, Leite MI, George JS, et al. The differential diagnosis of longitudinally extensive transverse myelitis. Mult Scler 2012;18: 271-85 CrossRef Medline

13. Yonezu T, Ito S, Mori M, et al. "Bright spotty lesions” on spinal magnetic resonance imaging differentiate neuromyelitis optica from multiple sclerosis. Mult Scler 2014;20:331-37 CrossRef Medline

14. Cobo-Calvo A, Alentorn A, Mañé Martinez MA, et al. Etiologic spectrum and prognosis of longitudinally extensive transverse myelopathies. Eur Neurol 2014;72:86-94 CrossRef Medline

15. Carnero Contentti E, Hryb JP, Diego A, et al. Etiologic spectrum and functional outcome of the acute inflammatory myelitis. Acta Neurol Belg 2017;117:507-13 CrossRef Medline

16. Gupta A, Kumar SN, Taly AB. Neurological and functional recovery in acute transverse myelitis patients with inpatient rehabilitation and magnetic resonance imaging correlates. Spinal Cord 2016;54: 804-08 CrossRef Medline 